\title{
SELECTED TOPICS IN THE GENERAL THEORY OF FUNCTIONS.
}

\author{
SIX LECTURES DELIVERED BEFORE THE CAMBRIDGE \\ COLLOQUIUM, AUGUST 22-27, 1898.
}

BY PROFESSOR W. F. OSGOOD.*

\section{Lecture $I$.}

Picard's Theorem, and the Application of Riemann's Geometric Methods in the General Theory of Functions.

THe subject which I have chosen for the first lecture of the Colloquium is Picard's noted theorem which in its more restricted form $\dagger$ may be stated as follows: Any function $G(z)$ which is single valued and analytic for all finite values of z takes on in general for at least one value of $z$ any arbitrarily assigned value $C$. There may be one value, a, which the function does not take on. But if there is a second such value, $b$, the function reduces to a constant.

To prove the theorem it is sufficient to establish the existence of a function $\omega(x)$ such that

(1) $\omega(x)$ is analytic for all but three values of $x$;

(2) $\omega(x)$ does not enter a certain region of the $\omega$-plane, no matter what path $x$ traces out in the $x$-plane.

For, let $x=G(z)$ and let the singular points of the function $\omega(x)$ be the points $a, b, \infty$. If $z$, starting with the value $z_{0}$, traces out a closed path in the $z$-plane, $x$, starting with the value $x_{0}$, will return to this value; but $\omega(x)$ may conceivably, when $x$ describes this path, fail to return to its original value; $i$. e., $x$ may have described a path which on the Riemann's surface of the function $\omega(x)$ is not closed. To show that this is not the case, Picard reflects that, the path in the $z$-plane being drawn together continuously to a point, the corresponding path in the $x$-plane must behave likewise and hence in the course of its deformation cannot pass over any one of the points $a, b, \infty$. Hence $\omega(x)$, regarded as a function of $z$, is a single valued function, analytic for all finite values of $z$. Now by a well known theorem of Weier-

* To Professors E. H. Moore and H. S. White for their assistance in editing these lectures for publication I wish to express grateful acknowledgments.

† Picard, "Sur une propriété des fonctions entières," Comptes Rendus, vol. 88 (1879); also his Traité d'Analyse, vol. 2, p. 231. 
strass's* such a function must for certain values of $z$ come arbitrarily near to any assigned value. If we choose as this value an interior point of the region in the $\omega$-plane that is never entered by $\omega$, we are led to a contradiction and the theorem is proved.

Thus far we have reproduced substantially Picard's reasoning. Let us notice that the proof is intuitional in the use it makes of continuous deformations of continuous curves. To place the proof on the firmest foundation we possess it remains to arithmetize these steps. It is entirely possible to do so, the method employed being substantially the same as that by which it is shown that the angle of a continuous function of a complex variable, be the function analytic or not, comes back to its original value when $z$ describes a closed path that forms the complete boundary of a region within and on the boundary of which the function does not vanish. I wish to call your attention, however, to a second method, also geometric, which is instructive, and which is fully as simple as the above.

Let $z_{0}$ be any value of $z$ and let $\omega_{0}$ be one of the values of $\omega\left(x_{0}\right)$, where $x_{0}=G\left(z_{0}\right)$. Then, since

and

$$
\omega-\omega_{0}=\tilde{q}_{1}\left(x-x_{0}\right)
$$

it follows that

$$
\begin{aligned}
& x-x_{0}=\text { 羪 }_{2}\left(z-z_{0}\right) \\
& \omega-\omega_{0}=\text { 㐿 }\left(z-z_{0}\right)
\end{aligned}
$$

and we wish to show that this last power series converges for all values of $z$. Suppose this were not the case. Let $z_{1}$ be one of the singular points of $\omega$, regarded as a function of $z$, on the circle of convergence of $q_{1}\left(z-z_{0}\right)$. Connect $z_{0}$ with $z_{1}$ by a right line. As $z$, starting from $z_{0}$, describes this line, $x$ describes a certain curve $L$ on the $x$-surface for $\omega(x)$ and the neighborhood of each point $z^{\prime}$ of the line goes over conformally upon the neighborhood of the corresponding point

\footnotetext{
*Weierstrass, "Zur Theorie der eindeutigen analytischen Functionen," $88 ;$ Abh. d. Berliner Akad. d. Wiss., 1876.

$\dagger$ I do not mean to imply that no geometric proof can be as rigorous as an arithmetic one. There is every reason to expect that geometry will sone time be placed on as rigorous a footing as that which arithmetic now possesses. But at the present time the most rigorous proofs which we have of theorems like the above are those which belong to that realm of geometry whose processes can be at once translated into arithmetic processes (i. e. arithmetized).

$¥$ Burkhardt uses the term arcus instead of the unfortunate one amplitude of a complex quantity, $a+b i$, to denote $\tan ^{-1} \frac{b}{a}$. It may well be rendered in English by angle.
} 
$x^{\prime}$ of $L$, or possibly, in the case of a finite number of points $z_{a}{ }^{\prime}$, upon a set of $p>1$ leaves spread out over the $x$-surface and connected at a branch point in $x_{a}{ }^{\prime}$. For simplicity, assume that no branch points $x_{a}{ }^{\prime}$ appear. Then it is possible to mark out a (narrow) strip in the $z$-plane containing the line $z_{0} z_{1}$ in its interior and related conformally to a strip in the $x$-plane containing the curve $L$ in its interior. But this latter strip is related conformally to a strip in the $\omega$-plane containing the curve corresponding to $L$. Thus the neighborhood of the point $z_{1}$ goes over conformally on the neighborhood of a point $x_{1}$ and the neighborhood of the latter point goes over in turn conformally on the neighborhood of a point $\omega_{1}$. Hence $\omega$, regarded as a function of $z$, is analytic in the point $z_{1}$, and this determination of $\omega$ in and near $z_{1}$ coincides for those values of $z$ near $z$ which lie within the original circle about $z_{0}$ with the values given by the series $\omega-\omega_{0}=$ gat $\left(z-z_{0}\right)$, since it can be obtained from that series by analytic continuation. This would be impossible if the point $z_{1}$ were a singular point of the function $\omega$ defined by the element

$$
\omega-\omega_{0}=\text { 策 }\left(z-z_{0}\right) \text {. }
$$

The excluded case of branch points in the points $x_{a}^{\prime}$ presents no difficulty.

This proof is, like the first proof, geometric. But one who is conversant with Weierstrass's theory of functions will observe that it can be arithmetized immediately by the elementary methods of that theory.

It may be remarked that analysis of the above nature is not infrequently useful in problems of elimination-problems too often treated superficially by formal methods.

It remains to construct the function $\omega(x)$. Picard obtained such a function by taking the ratio of the moduli of periodicity of an elliptic integral of the first kind

$$
\begin{array}{cc}
K=\int_{0}^{1} \frac{d x}{\sqrt{\left(1-x^{2}\right)\left(1-k^{2} x^{2}\right)}}, & K^{\prime}=\int_{0}^{1} \frac{d x}{\sqrt{\left(1-x^{2}\right)\left(1-k^{\prime 2} x^{2}\right)}} \\
k^{2}+k^{\prime 2}=1, & \omega\left(k^{2}\right)=\frac{2 K^{\prime} i}{4 K} .
\end{array}
$$

The proof that this function has the desired properties involves some development of the theory of the elliptic transcendents. Now I wish to set over against this method for obtaining $\omega(x)$ the general methods of Riemann's theory of functions,-methods which may with advantage be employed 
much more extensively than has hitherto been the case in the general theory of functions.

For this purpose I begin with a figure which in the geometry of inversion is a triangle each of whose angles is 0 , - the shaded triangle numbered (1). Let this triangle be

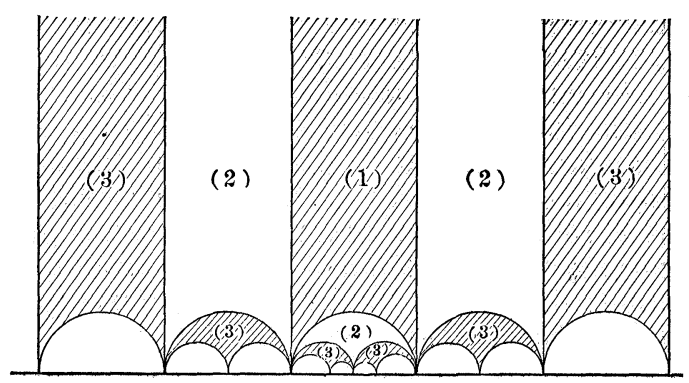

reflected* on each of its sides, thus generating the nonshaded triangles numbered (2). Again, reflect each of these new triangles on each of its outer boundaries, thus generating the shaded triangles numbered (3). And so on indefinitely. A figure is thus formed with the following properties :

(1) each pair of adjacent triangles are symmetrical to each other ;

(2) the triangles never enter the negative half-plane.

We are now in a position to construct the function $\omega(x)$. We may without loss of generality set $a=0, b \doteq 1$. This amounts to proving Picard's theorem for the function

$$
G_{1}(z)=\frac{G(z)-a}{b-a},
$$

whence the theorem for $G(z)$ follows. Riemann $\dagger$ enunci-

* By the image or reflection of a point $P$ in a circle is meant that point $P^{\prime}$ which lies on the line $O P$, on the same side of $O$ as $P$, and at such a distance from $O$ that $\overline{O P} \quad \overline{O P^{\prime}}=R^{2}$, where $R$ rlenotes the radius of the circle. A family of circles through $P$ and $P^{\prime}$ cuts the given circle orthogonally; and conversely, if a family of circles through two fixed points cuts a given circle orthogonally, the basis-points of the family are symmetrical to each other (i.e., mutual images of each other) with respect to the given circle. In any reflection of the plane, angles are preserved in magnitude, but reversed in sense, and circles go over into circles. Hence it follows that two points which are symmetrical to each other with respect to a given circle go over, when the plane is reflected in any other circle, into points symmetrical with respect to the transformed circle.

† Inaugural Dissertıtion, Göttingen, 1851 ; Gesammelte Werke, p. 40. 
ated the proposition that any simply connected region can be mapped conformally on a circle and Schwarz* gave a rigorous proof of it for the case that the boundary consists of a finite number of analytic curves. Let us map then the first triangle on the positive half of the $x$-plane, the vertices going over into the points $0,1, \infty$. If we denote the independent variable of the first plane by $\omega$, the map defines $\omega$ as an analytic function of $x$. This function can be continued across the axis of reals into the negative half-plane by Schwarz's principle of symmetry $\dagger$ as follows: Let $P$ be any point of the triangle (1), $Q$ the corresponding point in the positive half of the $x$-plane. Let $P^{\prime}$ be the image of $P$ in a specified side of the shaded triangle (1) (and hence a point of a specified one of the non-shaded triangles (2)), and let $Q^{\prime}$ be the image of $Q$ in the axis of reals. Thus the negative half-plane is mapped on the specified triangle (2). The function defined by this map is the analytic continuation of $\omega$ across that piece of the axis of reals which corresponds to the specified side of the triangle (1).

This process being repeated indefinitely, all the analytic continuations of the function $\omega(x)$ are obtained geometrically by reflections of the triangles of the $\omega$-plane in their sides. The points $0,1, \infty$ are singular points for each branch of the function $\omega(x)$, and they are the only singular points of this function.

The function $\omega(x)$ thus defined is the function sought.

We turn now to the more general form of Picard's theorem :

If $F(z)$ is any analytic function of $z$ which in the neighborhood of a point $A$ is single valued and has in this region no other singularities than poles, and if $A$ is an essentially singular point of $F(z)$, then there are at most two values which $F(z)$ does not take on in every neighborhood of the point $A$. If there are no poles in the neighborhood of $A$, there is at most one value that $F(z)$ does not take on in every neighborhood of $A$.

This theorem, it will be noticed, is concerned with the behavior of a function im Kleinen, i. e., throughout a certain arbitrarily small region; while the earlier theorem was one im Grossen, the domain of the independent variable being there the whole finite region of the plane.

The first part of the theorem follows from the second part. For, if $F(z)$ has poles clustering about $A$ and fails

* Cf. Picard, Traité d'Analyse, vol. 2, ch. 10.

† Ibid. 
to assume the value $a$, then the function $\frac{1}{F(z)-a}$ has no poles in the neighborhood of $z_{0}$ and hence by the second part of the theorem can fail to assume at most one value, which surely is not 0 . Denote this value by $\frac{1}{b-a}$. Then, if $c \neq a, b$, the equation

$$
\frac{1}{F(z)-a}=\frac{1}{c-a}
$$

can be satisfied, and hence $F(z)=c$, if $z$ is a root of that equation.

Let then $F(z)$ be analytic everywhere in the neighborhood of the point $A=\infty, i$. $e$, in the region $S$ lying outside of a certain large circle about $z=0$ as center ; and assume that, within $S, F(z)$ never takes on the values $a, b$, which we will again set equal to 0,1 . As in the earlier proof, let

$$
x=F(z) \text {. }
$$

Then if we cut $S$ along the positive axis of reals, $\omega(x)$, regarded as a function of $z$, will be single valued within this simply connected region $S^{\prime \prime}$. But when $z$ crosses the cut and, after encircling the point $z=\infty$, comes back to its original value, the path of $x$ may be an open path $\lambda$ on the Riemann's surface for $\omega(x)$ and $\omega$ will have gone over into a new value $\omega_{0}{ }^{\prime}$. Let us notice that in that case the whole neighborhood of $\omega_{0}$ will have gone over conformally into the neighborhood of $\omega_{0}^{\prime}$. In fact, this transformation of $\omega$, though defined originally by the paths in the $x$-plane and the $\omega$-plane which correspond to the path from $z_{0}$ back to $z_{0}$, may now be studied directly by means of the last two paths alone. But here $\omega^{\prime}$ appears simply as an analytic continuation of $\omega$ along a path in the $x$-plane. Such a continuation, we have seen, can always be obtained by reflecting the original triangle of the $\omega$-plane successively, and since $x$ has come back to its starting point, the reflections must be even in number ; for only then will shaded regions in the $\omega$-figure be carried over into shaded regions, non-shaded regions into non-shaded regions. And now I say : the transformation of the region about $\omega_{0}$ on the region about $\omega_{0}^{\prime}$ is a linear transformation of the complex variable $\omega$. For any reflection of the plane in a circle defines a transformation of the whole plane into itself which is conformal everywhere (including the point $\infty$ ) with reversal of angles. An even number of such reflections will define, then, a transformation of the whole plane into 
itself conformal everywhere without reversal of angles. Such a transformation defines an analytic function of a complex variable, and this function can readily be shown to be linear-a good exercise, by the way, for students in a course on the theory of functions. Hence

$$
\omega^{\prime}=\frac{\alpha \omega+\beta}{\gamma \omega+\delta} .
$$

Let $z$ describe the same path again. It follows. then, by a repetition of the above reasoning that $\omega^{\prime}$ will be carried over into a new branch $\omega^{\prime \prime}$, where $\omega^{\prime \prime}$ is a linear function of $\omega^{\prime}$. But will this be the same linear function as before? To answer this question we observe that the above linear relation between $\omega$ and $\omega^{\prime}$ holds, when $\omega, \omega^{\prime}$ are regarded as functions of $z$, for all values in the neighborhood of $z_{0}$. It must, therefore, continue to hold for all simultaneous analytic continuations of $\omega$ and $\omega^{\prime}$. And since the function $\omega^{\prime}$ can be continued analytically along the same path along which $\omega$ was just continued, it follows that at the end of the path

$$
\omega^{\prime \prime}=\frac{\alpha \omega^{\prime}+\beta}{\gamma \omega^{\prime}+\delta}
$$

Thus $\omega$, regarded as a function of $z$, is multiple valued, taking in each point of $S$ the values corresponding to the group of transformations consisting of the powers of the transformation

$$
\omega^{\prime}=\frac{\alpha \omega+\beta}{\gamma \omega+\delta} .
$$

We proceed now to form a function $\varphi(\omega)$ which shall be invariant by the transformations of this group, shall be a single valued function of $z$, and shall furthermore not enter every region of the $\varphi$-plane. To do this we will first show that the above linear transformation must be either

(a) the parabolic transformation

$$
\frac{1}{\omega^{\prime}-\omega^{(0)}}=\frac{1}{\omega-\omega^{(0)}}+k,
$$

in particular

$$
\omega^{\prime}=\omega+k,
$$

where $k$ is real ; or

(b) the hyperbolic transformation

$$
\frac{\omega^{\prime}-\omega^{(1)}}{\omega^{\prime}-\omega^{(0)}}=k \frac{\omega-\omega^{(1)}}{\omega-\omega^{(0)}},
$$

where $k$ is real, positive, and $\neq 1$. 
For, since each of the reflections used in generating the automorphic figure of the $\omega$-plane carries the upper and the lower half-planes over into themselves, the same must hold true of the linear transformation in question. Its fixed points must, therefore, either lie on the axis of reals, and in that case it will be one of the above transformations, or be symmetrically situated with reference to that axis. We proceed to show that this latter case is impossible.

The automorphic figure above generated covers the whole of the upper half-plane. For the purposes of the proof of this theorem it will be convenient to generate a new automorphic figure as follows, * and subsequently to show that it is identical with the old figure. Begin with the shaded

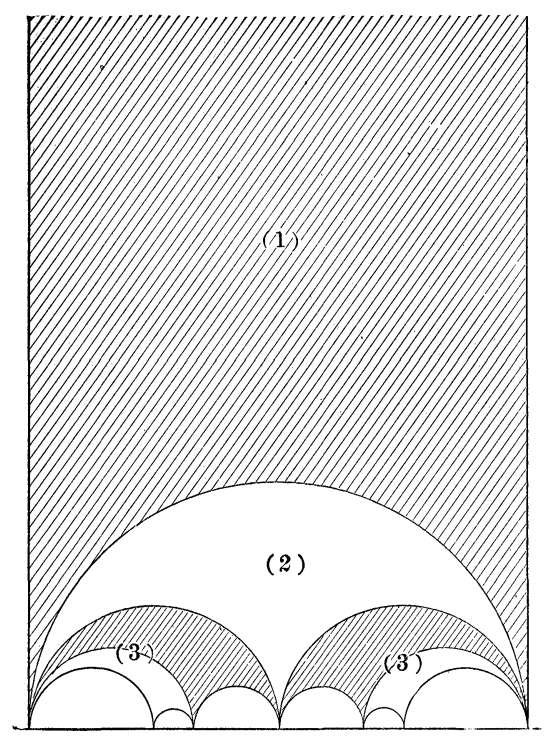

triangle (1) and reflect it on its semicircular side, thus generating the triangle (2). If the finite vertices of (1) were at the points 0 and 1 , a vertex of (2) will be at $1 / 2$. Now reflect the whole figure thus formed on each of the two semicircular boundaries, thus obtaining the triangles numbered (3). The maximum radius of a semicircle of the new boundary will be $1 / 2^{2}$. Next reflect the total figure thus formed in each semicircle of its boundary. The maximum radius of a semicircle of the new boundary will be $1 / 2^{3}$. And

* This method of proof was suggested to me by Professor Bôcher. 
so on. Hence the boundary of the figure will ultimately sweep over any preassigned point of the upper half-plane which lies between the parallels through 0 and 1. Finally, complete the automorphic figure by an indefinite repetition, in each direction, of the figure just constructed, interchanging, however, the shaded and non-shaded regions in such a manner that, in the complete figure, of any two adjacent triangles, one shall be shaded, the other non-shaded. Notice that, from the manner in which the figure was constructed, there can be no overlapping, not merely from step to step (im Kleinen), but after any series of steps (im Grossen).

It remains to prove that the two complete figures are identical. Each pair of adjacent triangles in the second figure are symmetric, either by construction or because they are the images of a symmetric pair. If then in the generation of the old figure we make the same shaded triangle (1) of the new figure the point of departure, as much of the old figure as at any given step has been generated will coincide throughout with a part of the new figure, and hence it can readily be shown $*$ that the old figure coincides throughout with the new one.

If now a fixed point $\omega^{(0)}$ of the linear transformation lay in the upper half-plane, we could connect $\omega^{(0)}$ with $\omega_{0}$ by a path lying in that half-plane, mark the corresponding path $x$ joining $x^{(0)}$ with $x_{0}$ on the Riemann's surface, add to this the path $\lambda$ above considered, and then add to $\lambda$ a path $x^{\prime}$ congruent to $x$, but lying, of course, in a different leaf. Let $\omega$, regarded as a function of $x$, be continued from $x^{(0)}$ along the complete path $x \lambda x^{\prime}$. Then $\omega$, starting at $x^{(0)}$ with the value $\omega^{(0)}$, will end at $x^{(0)}$ with the value $\omega^{\prime(0)}=\omega^{(0)}$. Hence the corresponding points of the $x$-surface $(i$.e., the extremities of the $x \lambda x^{\prime}$-path) must lie in the same leaf, and here is a contradiction.

We are now ready to form the function $\varphi(\omega)$. Begin with the case

$$
\omega^{\prime}=\omega+k .
$$

Then it is sufficient to set

$$
\varphi(\omega)=e^{\frac{2 \pi i}{|k|} \omega} .
$$

Since the pure imaginary part of $\omega$ is always positive,

$$
R\left(\frac{2 \pi i}{|k|} \omega\right)<0
$$

* The point is that the limiting figure approached in the generation of the old figure cannot be a part, but must be the whole of the new figure. 
where $R$ denotes the real part of the complex quantity. Hence

Similarly, if

$$
|\varphi(\omega)|=e^{R\left(\frac{2 \pi i}{|k|} \omega\right)}<1 .
$$

we may set

$$
\frac{1}{\omega^{\prime}-\omega^{(0)}}=\frac{1}{\omega-\omega^{(0)}}+k
$$

$$
\varphi(\omega)=e^{\frac{2 \pi i}{|\bar{k}| \frac{1}{\omega-\omega(0)}}}
$$

For the hyperbolic transformation $\varphi(\omega)$ may be defined as follows :

$$
\varphi(\omega)=e^{\frac{2 \pi i}{\mid \log k_{1}} \log \frac{\omega-\omega(1)}{\omega-\omega(0)}} .
$$

This function, to be sure, is not a single valued function of $\omega$; but it is a single valued function of $z$. For, $\omega$ remaining always in the positive half-plane, the angle of the complex quantity $\frac{\omega-\omega^{(1)}}{\omega-\omega^{(0)}}$ may be taken as lying between 0 and $\pi$, provided $\omega^{(0)}<\omega^{(1)}$, and hence the pure imaginary part of $\log \frac{\omega-\omega^{(1)}}{\omega-\omega^{(0)}}$ cannot vary by so much as $\pi i$. Thus $|\varphi(\omega)|$ is constrained to lie between two fixed limits. The proof is now complete.

The limitation of the linear transformation $\omega^{\prime}=\frac{\alpha \omega+\beta}{\gamma \omega+\delta}$ to the above two types could be established in various other ways. Thus it could be shown that, since this transformation carries one triangle over into a second, each triangle is carried over into a different one, and hence no point of the upper half-plane can be a fixed point. Or it could be shown directly that an even number of reflections of the kind here considered yield only parabolic and hyperbolic transformations of the above kind. But the complete discussion of the automorphic figure is valuable for other purposes. One property of this figure which can now be easily established has not explicitly come into play in the foregoing, namely, that a reflection on any side of any triangle carries the figure over into itself, except that shaded and non-shaded regions are interchanged.

It is hardly necessary to add that the methods set forth in this lecture are to a large extent those which Klein has done so much towards making a vital force in the modern theory 
of functions which Riemann initiated. Cf. Klein-Fricke, Modulfunctionen, vol. 1.

Another noted theorem of Picard's is the following:* If between two single valued analytic functions of a single variable there exists an algebraic relation of deficiency greater than unity, these functions cannot have an isolated essentially singular point. Picard published a proof in Darboux' Bulletin (1883), which however is incomplete in an essential respect. He gave two other proofs later in the Acta (1. c.), the first of which is based on properties of the automorphic functions. It would be interesting to apply the methods of this lecture to the proof of that theorem also.

\section{Lectures II and III.}

The Representation of Multiple Valued Functions by Means of Single Valued Functions of a Parameter, treated Geometrically by Riemann's Methods. Poincaré's Theorem.

Poincaré's Theorem. $\dagger$ Let $y=f(x)$ be any analytic function of $x$ whatsoever. Then it is possible to find two single valued analytic functions of a parameter $z$ such that, if

$$
\begin{aligned}
& x=\varphi(z), \\
& y=\psi(z),
\end{aligned}
$$

each value of $z$ pertaining to the domain of definition $T$ of the function $\varphi(z)$ will yield a point $(x, y) \$$ of the analytic configuration $y=f(x)$ and, conversely, to each point of this analytic configuration will correspond, in general, one or more values of $z$ pertaining to $T$, two such values of $z$ never becoming coincident. There will be at most three values of $x$ at which some branches of $f(x)$ are analytic and such that any point $(x, y)$ of the configuration in which one of these participates will fail of such representation. The domain $T$ will be the interior of the unit circle, or a domain included within that circle.

If, in particular, there are at least three values of $x$ which are singular points for all branches of the function, then the analytic configuration $y=f(x)$ is represented adequately by the functions $\varphi(z), \psi(z)$, in that each point $(x, y)$ of the configuration has cor-

* Picard, "Démonstration d'un théorème général sur les fonctions uniformes liées par une rélation algébrique"; Acta Math., vol. 11 (1888).

† Poincaré : "Sur un théorème de la théorie générale des fonctions ;" Bulletin de la Soc. math de France, vol. 11 (1883).

† This term is iıtended to express a grneralization of the familiar idea of a point of an algebraic configuration (Gebilde), and means a value of $x$ with which is coupled a value of the function $y=f(x)$ that belongs to a branch of that function which is analytic at $x$. 
responding to it at least one point $z$ of $T$, two such values of $z$ never becoming coincident. But even then the neighborhood of a branch point of finite order in the $x$-surface does not go over into the neighborhood of a point in $T$.

Before entering on the discussion of this theorem, let us consider some examples of representation by means of a parameter.

1. Suppose $f(x)$ is multiple valued and has two singular points. These may be thrown to 0 and $\infty$; and now two cases arise :

(a) $f(x)$ has but a finite number of values, $m$;

(b) $f(x)$ is infinitely multiple valued.

In the first case, the representation is effected by means of the equations

$$
\begin{gathered}
x=z^{m}, \\
y=f\left(z^{m}\right)=\psi(z) ;
\end{gathered}
$$

in the second case, by the equations

$$
\begin{gathered}
x=e^{z}, \\
y=f\left(e^{z}\right)=\psi(z) .
\end{gathered}
$$

2. A further example is furnished by the unicursal curves

$$
\begin{aligned}
& x=\varphi(z), \\
& y=\psi(z),
\end{aligned}
$$

where $\varphi(z), \psi(z)$ are rational functions of $z$, and $z=R(x, y)$.

In each of the above cases, to each value $z$ ( $T$ consisting of the whole plane with the exception of a finite number of points) corresponds in general a point $(x, y)$ of the analytic configuration, $y$ being in this point an analytic function of $x$; and, conversely, to each such point $(x, y)$ corresponds in general one, and never more than one, value of $z$. .

3. Next may be mentioned the representation of the coordinates of algebraic curves by the elliptic functions when $p=1$, and, generally, by automorphic* functions. Here the relation between $(x, y)$ and $z$ continues to be one-to-one $i m$ Kleinen, but is one-to-infinity im Grossen.

Turning now to Poincaré's investigations, we find that Poincaré begins by constructing a Riemann's surface for the function $f(x)$ in a novel manner. Let $x_{0}$ be a point at which $f(x)$ is analytic; let $a, b, c$ be any three values of $x$, intro-

* Cf., for example, Klein, Ueber lineare Differentialgleichungen der zweiten Ordnung 1894, (lithographed); in particular, p. 515, where Picard's "Methode des Linienelements" is set forth. 
duced for a purpose to be explained later, and let them be regarded as singular points of each leaf. Consider a path that starts from $x_{0}$ and returns to $x_{0}$. Such paths may be divided into two classes :

(a) paths that can be contracted to the point $x_{0}$ without sweeping over a singular point of the function or one of the points $a, b, c$;

(b) all other paths.

In the case of paths of the first class only shall the terminal point be said to belong to the same sheet of the Riemann's surface as the initial point.

Thesurface thusconstructed will be infinitely many leaved; it will be bounded by no other isolated singular points than branch points of infinitely high order, these points not being regarded, of course, as belonging to the surface; and thus the function $f(x)$ will be analytic at each point of the surface. Any closed curve on the surface can be drawn together continuously to a point, and thus the function $\int_{x_{0}}^{x} f(x) d x$ will be single valued on this surface.

Next, a set of curves containing $x_{0}$ is constructed bounding regions $C_{1}, C_{2}, \cdots$ such that each region is contained in each of its successors and each point of the Riemann's surface just constructed is ultimately contained in a $C_{n}$. That such a set of contours can always be constructed will be shown presently.

Poincaré then constructs for each $C_{n}$ the Green's function $u_{n}$ with its singularity in $x_{0}$ and shows that, $E$ being any region of the surface, $u_{n}$ converges uniformly on $E$

$$
\lim _{n=\infty} u_{n}=u \text {. }
$$

His analysis at this point is allied with that by which Harnack proved his noted theorem* that a function $u_{n}$ which is harmonic throughout aregion for all values of $n$, which always increases when $n$ increases, and which at one point of the region converges toward a limit, converges at all points within the region, and the limiting function is also harmonic. In fact, in the light of Harnack's theorem it is sufficient for Poincaré to show that the constantly increasing harmonic function $u_{n}$ converges toward a limit for one single point of the Riemann's surface,-and this he can do at once by means of his auxiliary function, which is virtually the same function $\omega(x)$ that we constructed in the previous lecture. $\mathrm{He}$

\footnotetext{
* Cf. Picard, Traité d'Analyse, vol. 2, p. 57.
} 
uses, moreover, only those properties of $\omega(x)$ that were deduced in the first part of that lecture. The three points $a$, $b, c$ are the singular points of the auxiliary function and were introduced solely for the purpose of proving the convergence of $u_{n}$.

Poincare then shows that the function

$$
z=e^{-(u+v i)},
$$

where $v$ denotes the conjugate function of $u$, maps the whole Riemann's surface either on the interior of the unit circle or on a region $T$ contained within it, which does not overlap itself. Thus $x$ appears as a single valued function of $z$; and $y$, being single valued on the surface, is likewise a single valued function of $z$. Notice, however, that while $\varphi(z)$ has a natural boundary, this is not necessarily the case for the function $\psi(z)$.

To show the possibility of constructing the contours, number the rational points of the Riemann's surface.* By a rational point $x=x^{\prime}+x^{\prime \prime} i$ is meant one for which $x^{\prime}$ and $x^{\prime \prime}$ are both rational numbers. Begin with those situated within the circle of convergence of the series

$$
f(x)=\text { 筙 }_{0}\left(x-x_{0}\right) \text {. }
$$

Put them down as the first line of a two-dimensional array (the sort of array used in writing out a doubly infinite series). Next, consider the continuations having these points as centers, and number their rational points (not already numbered), to begin with, in a two-dimensional array, the $n$th line giving the rational points in the circle of convergence about the rational point numbered $n$; then contract this array into a single line, the second line of the final array; - etc. $\dagger$ The final array can then be reduced to a single line. And now it is sufficient to take $C_{n}$ so as to include a circle about the point numbered $n$ whose radius is half the radius of the circle of convergence of the element whose center is that point. For, let $P$ be any point of the Riemann's surface, $i$. e., a point lying within the circle of convergence of an element g $_{1}\left(x-x_{1}\right)$ which is a continua-

\footnotetext{
* This is essentially what Poincaré did in numbering the elements (power series) defining the analytic continuation of $f(x)$, whose centers are rational points. Cf. Poincaré : "Sur une propriété des fonctions analytiques," Rendiconti del circolo matematico di Palermo, vol. 2 (1888).

t'The analysis here employed applies to the analytic continuation of any function whatsoever, an 1 thus fills a gap that frequently remains open in the presentation of the Weierstrassian theory of functions.
} 
tion of the element $g_{0}\left(x-x_{0}\right)$. Draw a circle about $P$ whose radius is one-third that of the radius of convergence of the series $f_{1}\left(x-x_{1}\right)$, and let $P^{\prime}$ be any rational point situated within this circle, the number of this point being $n$. Then $C_{n}$ will include the whole of this circle, and hence the point $P$.

In the foregoing, the points $a, b, c$ were introduced solely for the purpose of effecting the proof of convergence. Without them, the Riemann's surface, the contours $C_{n}$, and the Green's function $u_{n}$ could have been constructed as before. At a given point of the surface $u_{n}$ steadily increases as $n$ increases and if it could be shown that in one single point $u_{n}$ converges toward a limit, when $n=\infty$, we should be in possession of that generalization of Poincaré's theorem which consists in removing the restriction "in general." But it is not by the fact that certain points at which $f(x)$ is analytic (those, namely, for which $x=a, b, c$ ) or at which $f(x)$ has a simple singularity (a branch point of finite order, for example) have no corresponding point within $T$ that we are constrained to seek the essential limitations of Poincaré's theorem. An ideal theorem would be one by which the multiple leaved domain of definition of the function $f(x)$ is mapped in general conformally on a single leaved domain in the $z$-plane, the neighborhoods of certain isolated points (including isolated singularities and the point $x=\infty$ if necessary) going over by a well defined geometric law into the neighborhoods of vertices abutting on the boundary of $T$. Such a theorem exists for the algebraic functions, the auxiliary functions $\varphi(z), \psi(z)$ being automorphic functions with fundamental circle.

Poincaré's proof of the restricted theorem makes no use of other properties of the function $f(x)$ than the situation of the singularities of its various branches, and it thus establishes, in certain cases, the existence of functions belonging to a given Riemann's surface,-a question which for the most general Riemann's surface has not as yet been settled. The simplest case is that in which a simply connected Riemann's surface is given, with no other isolated singularities than branch points of infinite order, and such that at least three values of $x$ are included in no leaf. To such a surface there surely correspond functions.

A further point is the following: Nothing in Poincaré's analysis throws any light on whether the region $T$ fills the whole circle. Suppose that in certain cases it did not. Would there then exist a function $\varphi(z)$ whose domain of definition is the unit circle and which has otherwise the 
same properties as the old $\varphi(z)$ ? This question can be answered in the affirmative if the following theorem is true : The interior of any simply connected region whatsoever can be mapped conformally on the interior of a circle. The determination of the correctness or the incorrectness of this theorem would be a useful contribution to analysis.

Let us assume that this theorem is true. Then we can give with Klein* an elegant proof of the theorem that the coördinates of an irreducible algebraic curve can be represented as single valued automorphic functions, with fundamental circle, of a parameter $z$. For, construct Poincaré's surface for such a function $y=f(x)$. If now $x$ describes a path which, though not closed on the surface, nevertheless brings the element $\mathrm{y}_{0}\left(x-x_{0}\right)$ back into itself, we thus have a one-to-one and conformal transformation of the whole Riemann's surface into itself. This transformation defines then a similar transformation of the interior of the unit circle in the $z$-plane into itself, and such a transformation can readily be shown to be linear. Hence the function $\varphi(z)$ admits a group of linear transformations into itself and this group has the unit circle as fundamental circle, the generating transformations of the group being finite in number. The function $\varphi(z)$ is then an automorphic function with fundamental circle, and the same is true of $y=\psi(z)$.

Klein's reasoning is, however, of much wider scope than is here indicated. It applies to any function such that, if it is continued analytically along a path from $x_{0}$ back to $x_{0}$, the element $\mathrm{g}_{0}\left(x-x_{0}\right)$ comes back to its original value without the path's closing on Poincaré's surface.

The lecture closed with the suggestion of a line of thought which had for its object a possible test of the correctness of the first generalization of Poincaré's theorem above proposed.

\section{Lectures $I V$ and $V$.}

On some Recent Study of the Relation between the Properties of a Function defined by a Power Series and the Coefficients of that Series.

Let the constants $a_{0}, a_{1}, a_{2}$, etc., be given, and let a function be defined by the series

$$
F(x)=a_{0}+a_{1} x+a_{2} x^{2}+\cdots .
$$

The first question to be considered is of course that of con-

\footnotetext{
* 1. c. p. 519 et seq.
} 
vergence. Cauchy* in 1821 established a theorem, which can be stated best in terms introduced by G. Cantor. Represent in the usual manner by a set of points on a line the values $\left|a_{n}\right|^{\frac{1}{n}},(n=1,2,3, \cdots)$, and let the largest value belonging to a point of condensation $\dagger$ of the set be denoted by $A$; then if $A>0$, the series will converge when $|x|<\frac{1}{A}$ and diverge when $|x|>\frac{1}{A}$, while if $A=0$, the series will converge for all values of $x$. This gives as a necessary and sufficient condition for the convergence of the series for all values of $x$, that $\lim _{n=\infty}\left|a_{n}\right|^{\frac{1}{n}}=0$.

The foregoing theorem was obtained recently de novo by Hadamard + . He applies it to the further question : has the function defined by the series a singularity at a specified point upon the circle of convergence? The necessary and sufficient conditions for such singularity being deduced, it is possible at pleasure to produce series defining functions having singular points everywhere dense on their circle of convergence, and hence having this circle as a natural boundary. Such a function is given by the series $\Sigma \alpha_{\mu} x^{c} \mu$ if the further condition is satisfied :

$$
\frac{c_{\mu+1}-c_{\mu}}{c_{\mu}}>s>0
$$

for all $\S$ values of $\mu$ greater than some fixed value $\bar{\mu}$; that is, if with increasing $\mu$ the ratio $c_{\mu+1}: c_{\mu}$ becomes and remains greater than some quantity itself greater than unity.

A more general sufficient condition has been obtained by Fabryll, namely, the condition that $c_{\mu+1}-c_{\mu}$ shall increase without limit as $\mu$ increases. This and the former constitute sufficient conditions; necessary conditions also have been obtained, but only of a very general type.

\footnotetext{
* It is interesting to note that Cauchy here had the notion of a Cantor's set and of a derivative which might consist of more than one point.

† Analyse algébrique (Paris, 1821), pp. 59, 143, 151. Cf. also Résumés analytiques (Turin, 1833), p. 47.

¥ "Essai sur l'étude des fonctions données par leur développement de Taylor," thesis in Liouville's Journal, 4th series, vol. 8 (1892).

\& This condition, Hadamard points out, can be stated in somewhat more general form.

|| "Sur les points singuliers d'une fonction donnée par son développement et l'impossibilité du prolongement analytique dans des cas très généraux." Annales de l'École normale supérieure, 1896.
} 
Returning from this application, I wish next to call attention to the study of the simplest singularities of the function on the circle of convergence, namely poles. Darboux had given in $1878 *$ necessary conditions that the function represented by the series should have on the circle of convergence one pole of specified order, but no other singularity; and he had further considered the case of $p$ poles, and also of a branch point of finite order. The question of the sufficiency of his conditions Darboux did not raise, but Hadamard establishes it (1. c. \$14) for the case of a single pole of the first order. A simple pole occurs namely on the circle of convergence of the series

$$
a_{0}+a_{1} x+a_{2} x^{2}+\cdots
$$

when and only when two conditions are satisfied:

(a) $\frac{a_{\mu}}{a_{\mu+1}}$ approaches a finite limit, say $x_{1}$, for $\mu=\infty$;

$$
\left|\frac{a_{\mu}}{a_{\mu-1}}-\frac{1}{x_{1}}\right|^{\frac{1}{\mu}}
$$

becomes and remains, as $\mu$ increases, less than a quantity inferior to unity.

More complicated is his deduction of the precise conditions for the occurrence of poles of aggregate order $p$ and for the absence of other singularities. The notion of a Cantor's set plays here again an important rôle, as also in the problem of finding the zeros of a given power series. a problem which Hadamard solves by aid of the analysis that he has here developed.

In brief, the details of Hadamard's method are as follows. He first establishes a necessary condition that the function

$$
f(x)=a_{0}+a_{1} x+a_{2} x^{2}+\cdots
$$

have poles $x_{1}, x_{2}, \cdots$ of aggregate order $p$ on the circle of convergence of the series. Let

$$
\begin{aligned}
P_{p}(x) & =\left(1-\frac{x}{x_{1}}\right)^{\mu_{1}}\left(1-\frac{x}{x_{2}}\right)^{\mu_{2}} \cdots \\
& =1+A^{(1)} x+A^{(2)} x^{2}+\cdots+A^{(p)} x^{p} ; \\
P_{p}(x) f(x) & =\sum_{m=-p}^{\infty} b_{m} x^{m+p} .
\end{aligned}
$$

* "Mémoire sur l'approximation des fonctions de très-grands nombres, et sur une classe étendue de développements en série," Liouville's Journal, 3d series, vol. 4 (1878). 
Then

$$
\begin{gathered}
b_{m}=a_{m+p}+A^{(1)} a_{m+p-1}+\cdots+A^{(p)} a_{m}, \\
a_{-k}=0, k=1,2, \cdots, p .
\end{gathered}
$$

where

Let $\rho, \rho^{\prime}$ denote respectively the radii of the circles of convergence of the above series. $\rho^{\prime}$ is greater than $\rho$; then, if $\varepsilon$ denotes an arbitrarily small positive quantity,

$$
\left|a_{m}\right|<\left(\frac{1+\varepsilon}{\rho}\right)^{m} \quad \text { and } \quad\left|b_{m}\right|<\left(\frac{1+\varepsilon}{\rho^{\prime}}\right)^{m}
$$

for all values of $m>\bar{m}$ (a fixed integer). A function of the coefficients is now formed, defined by the determinant

$$
D_{m, p}=\left|\begin{array}{crrr}
a_{m} & a_{m+1} & \cdots & a_{m+p} \\
a_{m+1} a_{m+2} & \cdots & a_{m+p+1} \\
\cdots \cdots \cdots \cdots \cdots \cdots \cdots \cdots \cdots \cdots \cdots \cdots \cdots \cdots \cdots & \cdots \cdots \cdots \cdots \cdots \cdots \\
a_{m+p} & a_{m+p+1} & \cdots & a_{m+2 p}
\end{array}\right| .
$$

If the $i$ th column of this determinant, multiplied by $A^{(p+1-i)}$ $(i=1,2, \cdots, p)$, is added to the last column, the $a$ 's of that column will be replaced by $b$ 's of like index. Hence it follows that

$$
\left|D_{m, p}\right|<\left(\frac{1+\varepsilon}{\rho^{p} \rho^{\prime}}\right)^{m} \quad \text { or } \quad\left|D_{m, p}\right|^{\frac{1}{m}}<\frac{1+\varepsilon}{\rho^{p} \rho^{\prime}} .
$$

when $m$ exceeds a certain fixed value, and thus a necessary condition is obtained, which can be formulated as follows: Represent by a set of points the values $\left|D_{m, p}\right|^{\frac{1}{m}} ;$ then the largest value belonging to a point of condensation of the set is less than $1 / \rho^{p+1}$ and does not exceed $1 / \rho^{p} \rho^{\prime}$.

Conversely, consider the Cantor's set

$$
\left|D_{m, P}\right|^{\frac{1}{m}}, \quad(m=1,2, \cdots)
$$

for each value of $P: 1,2, \cdots$. The largest value $\lambda$ belonging to a point of condensation is surely not greater than $1 / \rho^{P+1}$. It may for some values of $P$ be less. Let $p$ be the smallest such value. Then $f(x)$ has no other singularities than poles on the circle of convergence, and their aggregate order is $p$. With the exception of these poles, $f(x)$ is analytic throughout a circle of radius $\rho^{\prime}$, where $\lambda=1 / \rho^{p} \rho^{\prime}$.

The proof of this theorem rests on the following reasoning. First the lemma is established that $\left|D_{m, p-1}\right|^{\frac{1}{m}}, m=\infty$, actually converges toward $1 / \rho^{p}$. Hence the $p$ equations which Hadamard introduces at this point 


$$
a_{m+p+i}+A_{m}^{(1)} a_{m+p-1+i}+A_{m}^{(2)} a_{m+p-2+i}+\cdots+A_{m}^{(p)} a_{m+i}=0
$$

will determine $p$ quantities $A_{m}^{(1)}, A_{m}^{(2)}, \cdots, A_{m}^{(p)}$ as functions of $m$. These quantities are shown to converge respectively towards limits $A^{(1)}, \cdots A^{(p)}$, when $m=\infty$, and the quantity

is such that

$$
b_{m}=a_{m+p}+A^{(1)} a_{m+p-1}+\cdots+A^{(p)} a_{m}
$$

$$
\left|b_{m}\right|<\left(\frac{1+\varepsilon}{\rho^{\prime}}\right)^{m}
$$

Thus if the polynomial

$$
P_{p}(x)=1+A^{(1)} x+A^{(2)} x^{2}+\cdots A^{(p)} x^{p}
$$

is formed and multiplied into $f(x)$, the product

$$
P_{p}(x) f(x)=\sum_{m=-p}^{\infty} b_{m} x^{m+p}
$$

will have a circle of convergence of radius $\rho^{\prime}>\rho$. This establishes the proposition.

Hadamard then proceeds to obtain the necessary and sufficient condition that $f(x)$ should have on the second circle, the circle of convergence of the $b$-series, only poles. He makes this determination directly, not by a mere repetition of the previous reasoning. And so on. The discussion of the results is easy. Perhaps one of the most striking consequences is the establishment of the necessary and sufficient condition that the function $f(x)$, defined by the power series $\sum_{m=0}^{\infty} a_{m} x^{m}$ and its continuations, should have no other singularities in the finite region of the plane than poles, and the determination of the position and order of each of these poles.

As an application of the foregoing Hadamard determines the zeros of a function, defined by a power series

$$
\psi(x)=C_{0}+C_{1} x+C_{2} x^{2}+\cdots,
$$

that lie within the circle of convergence of that series, explicitly in terms of the $C^{\prime}$ s.

Let

$$
f(x)=\frac{1}{\psi(x)}=a_{0}+a_{1} x+a_{2} x^{2}+\cdots
$$

Here $a_{m}$ can be expressed rationally in terms of $C_{0}, C_{1}, \ldots, C_{m}$. Next $D_{m, p}$ is expressed rationally in terms of the $C$, $\mathrm{s}$ and thus a solution of the problem, of a nature similar to that 
of the solution of the main problem above discussed, is obtained.

Daniel Bernoulli had pointed out that if one root $x_{1}$ of an algebraic equation is in absolute value smaller than any other root, it can be obtained as the limit when $m=\infty$ of a variable which is a rational function, for each value of $m$, of the coefficients of the equation, namely :

where

$$
\begin{gathered}
x_{1}=\lim _{m=\infty} \frac{s_{-m}}{s_{-m-1}}, \\
s_{i}=x_{1}^{i}+x_{2}^{i}+\cdots+x_{n}{ }^{i} .
\end{gathered}
$$

Runge* generalized Bernoulli's results. Hadamard's results amount to a still further generalization to power series.

It is worthy of note that the criteria occuring in the above theorems of Hadamard's differ from those usual in the lower analysis in that they do not turn upon the existence and character of a single limiting point, but rather upon those of a set of such points, namely the cluster points of a Cantor's set.

Two theorems of Hadamard's $\dagger$ next to be considered concern integral functions, whether rational or transcendental. As before, denote by $F(x)$ a function explicitly defined by the series

$$
F(x)=a_{0}+a_{1} x+a_{2} x^{2}+\cdots+a_{m} x^{m}+\cdots,
$$

and let the series converge for all finite values of $x$. The necessary and sufficient condition for this was seen to be that

$$
\lim _{m=\infty}\left|a_{m}\right|^{\frac{1}{m}}=0
$$

Of all points $x$ not lying without a circle of radius $R$ about the origin, there is at least one-call it $x$-upon the circumference, in which $|F(x)|$ attains the maximum value. That maximum value increases indefinitely with the radius $R$; but how rapidly? As to the manner of its increase Hadamard establishes the theorem: The absolute value of $F(x)$ for points $x,|x|=R$, becomes infinite less strongly than the expression

* Acta Math., vol. 6 (1885).

† "Étude sur les propriétés des fonctions entières et en particulier d'une fonction considérée par Riemann," Liouville's Journal, 4th series, vol. 9 (1893), p. 171 seq. 


$$
R^{\epsilon} \cdot e^{\int_{R_{0}}^{R} \frac{\psi(R)}{R} d R}
$$

where $\varepsilon$ is any arbitrarily small positive constant, and $\psi^{\prime}(R)$ is an increasing positive function of $R$ depending upon the coefficients $a_{0}, a_{1}, a_{2}, \cdots$ in the following manner. It is always possible to construct a positive continuous function* $\chi(\xi)$ such that

(a) if $a_{m} \neq 0$,

$$
\chi(m) \leqq \frac{1}{\left|a_{m}\right|},
$$$$
(m \geqq \bar{m}) ;
$$

(b) the function

$$
\eta=\varphi(\xi)=[\chi(\xi)]^{\frac{1}{\xi}}
$$

steadily increases as $\xi$ increases, and becomes infinite with $\xi$. The inverse of the function $\varphi$,

$$
\xi=\Psi(\eta),
$$

will then, for values of $\eta$ that exceed some fixed inferior limit $R_{0}$, be a single valued function of $\eta$, increasing indefinitely with $\eta$; and this is the function $\Psi$ whose definition completes the statement of the above theorem. In place of the particular $\Psi$ so defined we may substitute any increasing function which becomes infinite more strongly, and the theorem will hold true a fortiori.

An example is this: if from and after the value $m=\bar{m}$ the condition is satisfied

$$
\left|a_{m}\right| \leqq \frac{1}{(m !)^{a}}
$$

where $\alpha$ is some positive constant, then $|F(x)|$ becomes infinite less rapidly than $e^{R \beta}, R$ denoting $\mathrm{i} x \mid$ and $\beta$ being any constant greater than $\frac{1}{\alpha}$.

A second theorem given by Hadamard is based upon the lemma: if the real part of an integral function $G(x)$ is algebraically less than $R^{\lambda} \quad(R=|x|)$ for all values of $x$ whose $R$ exceerls some fixed inferior limit then $G(x)$ is a polynomial of degree not higher than $\lambda$. The theorem itself is as follows. If $F(x)=e^{G(x)}$, where $G(x)$ denotes an integral function, and if for all values of $x$ having $|x|=R>\bar{R}$ the absolute value of $F(x)$ is less than $e^{R^{\lambda}}$, then $G(x)$ is a polynomial of de-

* Harlamard's analysis at this part of his memoir requires revision. The results appear to be correct. The account that I gave in the lecture of the construction of the function $\chi(\xi)$ must be essentially modified in details, but the general method was, I believe, correct. 
gree not higher than $\lambda$. It may be remarked in passing that for such integral functions $F(x)$ Picard's theorem is at once seen to be true, since the equation

$$
e^{G(x)}=c \neq 0,
$$

where $c$ is arbitrary and $G$ is a polynomial, always has roots.

The third theorem to which we now turn is the great theorem of this paper. It relates to the zeros of an integral function. Denote by $\rho$ the absolute value of any zero $x_{p}$ of the integral function

$$
F(x)=\sum_{m=0}^{\infty} a_{m} x^{m}
$$

and arrange the zeros in a definite order, giving to each the corresponding index $p$ in such a manner that always $\rho_{p+1} \geqq \rho_{p}$. If a function $\varphi(m)$, monotonic when $m>\bar{m}$, be so chosen that

$$
\frac{1}{\varphi(m)} \geqq\left|a_{m}\right|^{\frac{1}{m}}, \quad(m>\bar{m}),
$$

then, for any arbitrarily small positive quantity $\varepsilon$, an inferior limit $\bar{p}$ can be found such that, for $p>\bar{p}$,

$$
\rho_{p}>(1-\varepsilon) \cdot \varphi(p) \text {. }
$$

An illustration of this third theorem is given in any series having

$$
\left|a_{m}\right| \leqq \frac{1}{(m !)^{a}} .
$$

Assuming any $\alpha^{\prime}$ such that

and taking

$$
0<\alpha^{\prime}<\alpha,
$$

$$
\varphi(m)=m^{a^{\prime}},
$$

there results $\rho_{p}>p^{a^{\prime}}$, and the genre* of $F(x)$ is an integer not exceeding $\frac{1}{\alpha}$.

The impulse to the inquiries culminating in these theorems was given by two theorems of Poincaré, $\uparrow$ inspired by the researches of Weierstrass \$:

*A term introduced by Laguerre, Comptes Rendus, vol. 94 (1882), p. 160. Cf. Forsyth's Theory of Functions, \&. 59 et seq.

†"Sur les fonctions entières :" Bulletin de la Soc. math. de France, vol. 11 (i883)

$\ddagger$ "Zur Theorie der eindeutigen analytischen Functionen;" Abh.d. Berliner Akad., 1876 ; Math. Werke, vol. 2, p. 7. 
(a) If $F(x)$ has the genre $N$, then

$$
\text { I } F(x)<k \cdot e^{c R^{N+1}}
$$

for all values of $x$ having $|x|=R>\bar{R}$, where $k, c$ denote any real positive constants.

$$
\begin{gathered}
\quad(b) \text { If } \quad F(x)=\sum_{m=0}^{\infty} a_{m} x^{m} \quad \text { has the genre } N \text {, then } \\
\left|a_{m}\right|<\frac{\varepsilon}{(m !)^{\frac{1}{N+1}}}
\end{gathered}
$$

where $\varepsilon$ is taken arbitrarily small, provided $m$ is sufficiently large.

Poincaré also raised certain questions regarding the genre of sums and derivatives of functions whose genre is given. Concerning Poincaré's two theorems Hadamard shows that with slight modifications the converse theorems are true.

Borel has studied a number of questions akin to those that we have here considered, and has carried further some of the investigations instituted by Hadamard. I refer to his memoir in the Acta Mathematica, vol. 20 (1897): "Sur les zéros des fonctions entières."

Hadamard closes his memoir with a valuable application of the results he has obtained to the study of Riemann's function $\zeta(s)$.

\section{Lecture VI.}

On certain Cantor's Sets, and their Application in a Question concerning Cauchy's Definition of an Analytic Function.

In investigations in the general theory of functions, either of real or of complex variables, a clear insight into the possibilities that Cantor's sets present is indispensable. It is here less a question of systematic theoretical developments than of a body of knowledge of facts given by concrete examples. I should like to present to you today one or two examples that I have frequently found useful in work of a general nature.*

* The last of these examples is, for instance, useful in forming a critique of the classification of Cantor's sets which Painlevé adopts (Cf. his Thesis: "Sur les lignes singulières des fonctions analytiques," Paris, 1887; Toulouse Annales, vol. 2 (1888)). The division into the three classes, ponctuel, linérire, superficiel suggests that a set which is ponctuel, for example, is a fortiori, linéaire, and for that reason is excluded from that class. That would surely not be the case if Painleve had used the square instead of the circle in defining ponctuel, as the last of my examples shows. If on the other hand, the set of that example is in fact not ponctuel, then it fails to fall under any one of the three classes. 
I will begin with a set of points on a line, the conception of which enabled me to give the first example,* so far as I know, of a series of real terms

$$
u_{1}(x)+u_{2}(x)+\cdots
$$

of the following sort: for each value of $x$ in the interval $a \leqq x \leqq b$ each term of the series is continuous and the series converges toward a continuous function $f(x)$; the term-byterm integral

$$
\int_{x_{0}}^{x} u_{1}(x) d x+\int_{x_{0}}^{x} u_{2}(x) d x+\cdots
$$

likewise converges toward a continuous function, $\varphi(x)$; but

$$
\varphi(x)+\int_{x_{0}}^{x} f(x) d x .
$$

This set is a special case of a set given by Harnack $\dagger$ and is constructed as follows. It shall lie in an interval $L$ of unit length.

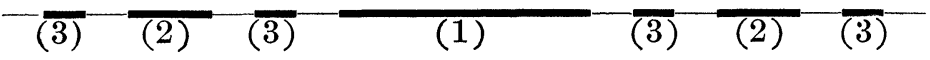

First Step. In the middle of this interval lay off an interval (1) of length

$$
l_{1}=\lambda-\frac{1}{3} \lambda,
$$

where $\lambda$ is chosen arbitrarily as a positive quantity not greater than the unity : $0<\lambda \leqq 1$.

In particular, let $\lambda=\frac{3}{4} ; l_{1}=\frac{1}{2}$.

Second Step. In the middle of each of the free end-intervals lay off an interval (2), both of these intervals to be of the same length $l_{2}$ and such that the total length of the intervals $(1),(2)$ is

$$
l_{1}+2 l_{2}=\lambda-\frac{1}{4} \lambda \text {. }
$$

$n$-th Step. In the middle of each of the equal free intervals lay off an interval $(n)$, all of these intervals to be of the same length $l_{n}$ and such that the total length of the intervals $(1),(2), \cdots,(n)$ is

$$
l_{1}+2 l_{2}+2^{2} l_{3}+\cdots+2^{n-1} l_{n}=\lambda-\frac{1}{n+2} \lambda .
$$

* Cf. \& 20 of my paper : "Non-uniform convergence and the integration of series term by term," Amer. Jour. of Math., vol. 19 (1897).

† Math. Ann., vol. 19, p. 239. 
When $n$ increases indefinitely, a set of intervals is obtained and their extremities form a Cantor's set. This set is enumerable. It is not perfect, $i$. e., does not coincide with its derivative. Let all the limiting points not already included in the set be annexed. Then we have a perfect set, and this set is the first example I wish to show you.

The content* of this set is $1-\lambda$ and hence is not, in general, 0 .

In order to obtain an example of a two-dimensional set which is nowhere dense in two dimensions, $i$. $e$, is such that in every neighborhood of any point of the set there exist two-dimensional regions which are free from points of the set, it is sufficient merely to erect at each point of the above set a perpendicular of unit length to the line on which the set lies, all the perpendiculars being drawn on the same side of this line. But it not infrequently happens that the simplest example is not the most striking one. And so here. You would prefer to see a set of points containing no lines. Very well. Take the perpendicular at one extremity of $L$ as a new interval $L^{\prime}$ and construct in $L^{\prime}$ a set of points like the set just constructed in $L$. Through each point of this set draw a parallel to $L$; the points of intersection of these parallels with the above perpendiculars form the set in question.

It is readily shown that the content of this set is $(1-\lambda)^{2}$, and is then in general not 0 . Moreover, if $\varepsilon$ is an arbitrarily small positive quantity and $P$ any point of this set, a rectangle can obviously be drawn containing $P$, but having no points of the set on its boundary, and having its sides less than $\varepsilon$ in length.

As an instance of the use of such conceptions of Cantor's sets as those just considered may be mentioned a question that arises in the Cauchy definition of an analytic function. Let $f(z)$ be a single valued function (the word function being used here in its most general sense), defined at each point of a two-dimensional region $T$, and let it have, at each point of $T$, a finite derivative, $i$. e., for any such point $z$, let

$$
\frac{f(z+h)-f(z)}{h}
$$

\footnotetext{
* Amer. Jour., 1. c. $\xi 17$. Let the interval $L$ be divided into $1 / 2^{m}$ equal parts and denote the sum of those parts to which points of the set pertain by $s_{m}$. Then the content of the set is defined as $\lim _{m=\infty} s_{m}$. The extension of the definition to two-dimensional sets is at once obvious, the above subintervals being replaced by squares $1 / 2^{m}$ on a side ; etc.
} 
converge toward a finite limit $f^{\prime}(z)$ when $h$ approaches 0 . What next? It is customary to add the condition that $f^{\prime}(z)$ be continuous in $T$; for without this condition no proof of Cauchy's integral theorem has as yet been given. Now the question is' whether this further condition is not superfluous; $i$. e., whether the continuity of $f^{\prime}(z)$ does not follow from the preceding conditions, or at least from those coupled with a less comprehensive condition; as for example, that $f^{\prime}(z)$ remain finite in $T$

$$
\left|f^{\prime}(z)\right|<G,
$$

where $G$ is some fixed quantity. That a little less than the assumption of exceptionless continuity will suffice is easily seen. For, from the existence of the derivative follows the continuity of the function $f(x)$. Now it is both a necessary and a sufficient condition for the continuity of $f^{\prime}(z)$ that $\int f(z) d z$, taken along any closed path in $T$ that can be drawn together continuously to a point of $T$, should vanish.* Hence it is readily shown that if $f^{\prime}(z)$ is in general continuous in $T$, but behaves in an unknown manner at isolated points or along curves that are not too crinkly, then $f^{\prime}(z)$ will be continuous everywhere in $T$. We can go a step further and state the theorem : If $f^{\prime}(z)$ is in general continuous in $T$, the points about which no supposition is made forming a set of content null, and if

$$
\left|f^{\prime}(z)\right|<G,
$$

where $G$ is a fixed quantity, then $f^{\prime}(z)$ is everywhere continuous in $T . \dagger$ For Goursat's proof of Cauchy's integral theorem applies, with a slight modification, to this case.

* Cf. "Some points in the elements of the theory of functions," this BULLETIN, 2d Ser., vol. 2, 1895-96. At that time a paper by G. Morera "Un teorema fondamentale nella teorica delle funzioni di una variable complessa," Real. Ist. Lomb. Kend. (2) vol. 19, Apr., 1886, was unknown to $m e$, in which the author not only establishes the ahove condition as sufficient, but applies his results to prove Weierstrass's theorem that a series of functions, analy tic throughout a two-dimen-ional region, which converges uniformly within this region, defines a function analytic within the region.

+ Professor Chessin states this theorem without the restriction that $\left|f^{\prime}(z)\right| \div G$. (Cf. Annals of Math., vol. 11, 1896.) His proof, which is given by a familiar method, is based on the following theorem (1. c., pp. 52, 53), which he ascribes to Harnack, but which Harnack neither states nor proves: Let $X(x, y)$ and $Y(x, y)$ be two functions of the real variables $x$ and $y$, which are finite and continuous throughout a connected domain $(I))$, and which generally ( $i$. e., with the exception of points and lines forming a discrete multiplicity) satisfy the equation $\frac{\partial X}{\partial y}=\frac{\partial Y}{\partial x}$; let further $\left(x_{0}, y_{0}\right)$ 
Finally, we may add the following theorem, which includes the preceding one as a special case : If $f^{\prime}(z)$ exists at each point of $T$ and if $\frac{\partial u}{\partial x}, \frac{\partial u}{\partial y}$ (where $f(z)=u+v i$ ) are each capable of surface integration over any two dimensional region contained in $T$, as well as of partial integration with regard to $x$ and $y$ (i.e., line integration parallel to either axis), then $f(z)$ is analytic in $T$.

For $u$ and $v$ are continuous functions of $x$ and $y$ in $T$, satisfying the relations

$$
\frac{\partial u}{\partial x}=\frac{\partial v}{\partial y}, \quad \frac{\partial u}{\partial y}=-\frac{\partial v}{\partial x}
$$

each of the integrals

$$
\int(u d x-v d y), \quad \int(v d x+u d y)
$$

taken along a closed path that cuts out from $T$ a simply connected region, can be shown by Green's theorem* to vanish ; and hence $\int f(z) d z$, taken along such a path, vanishes too.

This theorem is in one respect more general than any of the preceding in that it makes no supposition about the continuity of $f^{\prime}(z)$ whatsoever. It meets its limitations, however, already in the case that, $f^{\prime}(z)$ being in general continuous in $T$, nothing is known about the behavior of $f^{\prime}(z)$ in the points of a Cantor's set of positive content, -for example, the above set when $\lambda<1$.

It may be remarked that the existence of the derivative $f^{\prime}(z)$ involves more than the mere existence of the partial derivatives of $u$ and $v$, and of the relations between them.

and $(x, y)$ be any two points within $(D)$; then the definite integral

$$
\int_{\left(x_{0}, y_{0}\right)}^{(x, y)}(X d x+Y d y)
$$

will be independent of the path of integration, provided the several paths lie entirely within $(D)$ and can be brought to coincide with one another by a continuous deformation without crossing any of the boundary lines of $(D)$. Without introducing further restrictions there is at present no prospect of obtaining a proof of this theorem (if indeed it be true).

* The proof of Green's theorem in the generality here required depends on the following theorem : If $\phi(x)$ is defined for each value of $x$ in the interval : $a \leqq x \leqq b$, and if $\phi(x)$ has in each of these points a finite derivative $\phi^{\prime}(x)$; if furthermore $\phi^{\prime}(x)$ is integrable in the interval, then

$$
\int_{x_{0}} \phi^{\prime}(x) d x=\phi(x)-\phi\left(x_{0}\right)
$$


It involves the existence of a complete differential of the functions $u$ and $v$. Cf. Stolz, Differential und Integralrechnung, vol. 1 , ch. $4, \S 8$ and vol. 2 , ch. $12, \S 7$.

To test the truth of a general theorem one mode of procedure is to form an example which proves the theorem to be false. From the foregoing it appears that in the present case simpler examples are surely impossible than those

(a) in which $f^{\prime}(z)$ becomes discontinuous along a curve whose tangent does not turn continuously along any arc ; $*$

(b) in which, $f^{\prime}(z)$ being assumed to remain finite in $T$, $f^{\prime}(z)$ becomes discontinuous in a Cantor's set of positive content ;

(c) in which not both of the partial derivatives $\frac{\partial u}{\partial x}, \frac{\partial u}{\partial y}$ are capable of surface integration over any region lying in $T$, or of line integration along a line parallel to one of the coördinate axes.

HARVARD UNIVERSITY, September, 1898.

\section{THE FIFTIETH ANNIVERSARY MEETING OF THE AMERICAN ASSOCIATION FOR THE ADVANCEMENT OF SCIENCE.}

The semi-centennial meeting of the American Association for the Advancement of Science was held in Boston, August 22-27. There were over 900 members and associates registered. A large number of members of affiliated societies were also in attendance, many of whom did not register, but had the privilege of taking part in the proceedings of the sections in which they were interested. Their presence increased the general scientific interest; and it was the evident desire of the various sections to have still closer relations with the respective affiliated societies, as it is felt that any tendency towards the isolation of groups of specialists may partially defeat one of the objects the Association has at heart, viz., the spread of a popular interest in the work of scientific men.

The section of mathematics and astronomy, and its near neighbor, the physical section, were well attended, the

* It is possible that by analysis similar to Jordan's, Cours d'Analyse, vol. $1, \& \& 193-196$, this condition may be made more general. 